\title{
When Life Happens: Investigating Short and Long-Term Effects of Life Stressors on Life Satisfaction in a Large Sample of Norwegian Mothers
}

Gunvor Marie Dyrdal, $1 \bowtie$

Phone (+47) 90175356

Email gunvor.dyrdal@ntnu.no

Espen Røysamb, 2,3

Phone (+47) 94295177

Email espen.roysamb@psykologi.uio.no

Ragnhild Bang Nes, 2,3

Phone (+47) 21078249

Email Ragnhild.Bang.Nes@fhi.no

Joar Vittersø, 4

Email joar.vitterso@uit.no

1 Norwegian University of Science and Technology in Gjøvik, Gjøvik, Norway

2 Department of Psychology, University of Oslo, Oslo, Norway

3 Norwegian Institute of Public Health, Oslo, Norway

4 Department of Psychology, University of Tromsø, Tromsø, Norway

\section{Abstract}

The purpose of this study was to examine the impact of major life stressors on the short and long-term life satisfaction (LS) of Norwegian mothers using 
data from the Norwegian Mother and Child Cohort study (MoBa, N = 46,342). Data on LS were collected at T1 (6 months postpartum) and T3 (36 months postpartum), and data on life stressors at T2 (18 months postpartum) and T3. Altogether, 24,216 participants reported life stressors between $\mathrm{T} 1$ and $\mathrm{T} 2$, and 25,284 between T2 and T3. Life stressors had significant negative short-term and long-term effects on LS. Experiencing multiple stressors increased the negative impact on satisfaction linearly. Relationship dissolution, economic problems, becoming seriously ill, and conflict with family/friends most strongly predicted short-term LS (Cohen's $d$ -.18 to -1.15). Being pressured to sexual acts, relationship dissolution, economic problems and becoming seriously ill most strongly predicted longterm LS (Cohen's $d-.15$ to -1.05 ). When calculating the overall societal burden of life stressors, economic problems, conflict with family/friends, and work-related problems were shown to be particularly detrimental to maternal life satisfaction.

\section{Keywords}

Life satisfaction

Life events

Stressors

Short-term

Long-term

Wellbeing

Societal burden

The Norwegian Mother and Child Cohort Study (MoBa)

\section{Introduction}

Entering parenthood by having a baby is commonly associated with excitement, curiosity, and joy about the newborn child and what is to come. A period of elation lasting 4-6 weeks following childbirth has been observed, and is often referred to as the "baby honeymoon" period (Hobbs 1968, p. 414). Yet, this 
period is also a vulnerable phase in life, not only for mothers, but also for the child and the forming family, and hence, for a new generation. The transitional phase following childbirth entails an ability in parents to adjust to new circumstances, demands, and needs, and is found to be associated with increased personal and marital stress (e.g., Miller and Sollie 1980). Couples often seek professional help during this life phase, and effort has been made to identify factors that facilitate and inhibit the transition, in order to enable health professionals to better support parents in need (e.g., Barimani et al. 2017). Experiencing adverse life events, or stressors, during this challenging, transitional phase, may exaggerate parental pressures. It may also potentially have negative long-term consequences-not only for the parents, but for the future health and functioning of the child and the forming family.

$\mathrm{AQ} 4$

There is a considerable literature on the negative impact of mothers' stress, anxiety, and depression on the development, health, and future wellbeing of their unborn child (e.g., Glover 2011, 2014). Much less is known about how mothers' satisfaction with life is affected by stressful events during the early phases of parenthood. In general, negative life events or life stressors have been associated with mental health problems and reduced wellbeing (e.g., Burns and Machin 2013; Luhmann et al. 2011; Charles et al. 2013; Nes et al. 2014a, b), and to heightened risk of negative life outcomes, like bankruptcy (Maroto 2015). It is important to document the impact of life stressors on wellbeing and to understand their effect at vulnerable stages in life, in order to prevent detrimental outcomes or provide better support to those who are exposed.

The term subjective wellbeing includes both a cognitive evaluation of life as well as an affective component. Measures of life satisfaction are tapping the cognitive evaluation, while the term happiness commonly refers to the affective component. However, the constructs partly overlap. The results of numerous studies suggest that subjective wellbeing is relatively stable over time (for reviews, see Lucas 2018; Luhmann et al. 2011). According to the adaptation model of happiness (Brickman and Campbell 1971), which has influenced wellbeing theory considerably, most people return to an individual set-point, or personal satisfaction baseline, following major life events. Personality and genetic factors are shown to contribute substantially to this stability (Diener and Lucas 1999; Nes et al. 2006). These findings are also in accordance with the 
"dynamic equilibrium model" proposed by Headey and Wearing (1989).

According to the "dynamic equilibrium model", life events produce mainly temporary changes in wellbeing. However, evidence indicates that complete adaptation does not always occur and that wellbeing is subject to change (e.g., Dyrdal et al. 2011; Infurna et al. 2016b; Lucas 2005, 2007). This contradictory evidence has led former "adaptationists" to revise their views (e.g., Headey 2013; Luhmann et al. 2011).

Life challenges can take many forms, and researchers have attempted to differentiate between different types of stressors, such as formative life events, minor hassles, but also positive events like daily uplifts (Kanner et al. 1981). Different types of stressors influence people differently, both in terms of duration and in the magnitude of their effects. In general, most people tend to show a remarkable ability to adapt and thrive even after extremely difficult conditions (e.g., Bonanno 2004). For some individuals, intense turmoil and trauma might even lead to psychological transformation, response shift, or benefit finding (Seery et al. 2010; Taylor 2012). Yet, little is known about how different stressors influence mothers in their child-rearing years, and particularly about possible long-lasting effects. Most existing studies have tended to employ cross-sectional designs, and while a few longitudinal studies exist (e.g., Infurna et al. 2016a), these studies tend to examine the effects of only one or a few stressors concurrently (e.g., Lucas 2005, 2007). The current study aims to address this gap by examining the effect of single versus multiple stressors on satisfaction with life in mothers. The ten stressors examined were divorce, financial problems, death of someone close, problems at work, conflict with family, friends, or neighbors, pressured to sexual acts, personal illness/injury, illness/injury among close relatives, serious accidents, and worries about the child. Using a large population-based longitudinal sample of mothers, we explored the effect of these ten life stressors on both short- and long-term life satisfaction. Our research design allowed us to control for initial life satisfaction levels, thereby providing evidence of possible causal effects. The large sample also allowed us to explore the prevalence of different stressors among mothers, and to compare effects on the individual and on the group or population level. Hence, while some stressors may have strong short-term effects on satisfaction, their long-term impact may be modest. Other stressors may have dramatic effects, but if they are occurring less frequently, their overall 
population-level impact may be modest. Yet other stressors may have only modest or moderate individual effects, but be highly prevalent. Thus, they may yield large reductions in overall population, or societal, life satisfaction. Hence, in addition to addressing the cost of life stressors on the individual, we calculated an estimate of the societal impact of the stressors using a population attributable risk estimate. This allowed us to explore not only how life stressors affect the overall life satisfaction of mothers in the years they have children and create a family, but also how stressors impact life satisfaction on a societal level. A mother's experiences, dissatisfactions, worries, and life circumstances impact not only herself, but also her partner, family, and children, profoundly. Hence, the societal impact estimate, while based on data from mothers in our sample, may be taken as an indicator of overall population effects.

\section{Wellbeing and Areas of Life}

Subjective wellbeing is a broad concept that reflects how people subjectively evaluate their lives, and is most often measured as overall life satisfaction (e.g., Diener 1994; Diener et al. 2003, 2017). Wellbeing constitutes an important goal for individuals and societies with far-reaching positive consequences not only for population health (Howell et al. 2007) and longevity (e.g., Diener and Chan 2011), but also for personal growth (Fredrickson 2013), social relationships (Diener and Seligman 2002), and successes in the workplace (Boehm and Lyubomirsky 2008). The wellbeing and health of mothers may be of particular significance, as it may not only affects the mother herself but also the way she interacts with her family and the environment.

\subsection{Social Relationships}

Positive interpersonal contact and social relationships are essential for healthy human functioning (e.g., Ainsworth 1991; Diener and Tay 2015; Oishi 2012). Both cross-sectional and longitudinal evidence have quite consistently shown a significant positive association between wellbeing and indicators of social relationships, such as marriage (Lucas and Clark 2006), frequency of contact with family and friends (Powdthavee 2008), family social support (North et al. 2008), the number of one's social identities (Thoits 1983), and social networks (Coviello et al. 2014). Hence, substantial evidence supports the positive association between wellbeing and indicators of social ties. 
By contrast, loneliness (Cacioppo et al. 2008) and loss of social ties, through relationship dissolution (Rhoades et al. 2011), divorce (Lucas 2005), or widowhood (Ben-Zur 2012), are found to have a negative impact on life and satisfaction levels. Not only does divorcing your spouse significantly reduce life satisfaction, it is also associated with increased psychological distress (Rhoades et al. 2011). While the positive effect of relationship formation (i.e., marriage) seems to be mainly transient (Lucas and Clark 2006), the loss of a partner tends to have long-term negative effects on life satisfaction (Ben-Zur 2012; Lucas 2005). The experience of losing a close relationship, or the death of someone close during pregnancy or early parenthood, may have particularly negative consequences. Previous researchers have also shown that the relationship to one's partner is of particular importance for mothers' life satisfaction during this particular phase of life (Dyrdal et al. 2011).

Experiencing conflict in social relationships generates stress for most people. Most people are dependent on others for maintaining health and functioning, so when our social relationships suffer, we also tend to suffer (e.g., Umberson and Montez 2010). Indeed, social relationships are so important that they may even influence our mortality risk (Holt-Lunstad et al. 2010).

\subsection{Work}

Most people spend a considerable amount of time at work. Hence their job and work-environment are greatly influential and constitute an important part of life (Robinson et al. 2012; Warr 1999). Employment provides important benefits over and above its financial aspects, such as appreciation and social inclusion, a sense of purpose and belonging, professional advancement, engagement, autonomy, personal growth, and self-esteem (e.g., Goldsmith et al. 1996). Substantial literature also suggests that having multiple roles, like that of mother and employee, is beneficial ("role enhancement hypothesis"; Barnett and Hyde 2001). Additionally, employment provides a number of health-related benefits for mothers (Nes et al. 2006, 2014a; Rosenzweig et al. 2008), including even those who do not desire paid employment (Usdansky et al. 2012). Leaving or losing a job may therefore have far-reaching and negative long-term consequences for mothers' health and well-being (Ahrens and Ryff 2006; Holmes et al. 2012), and may also affect their child (McMunn et al. 2010). 
Conflict at work may likewise compromise maternal wellbeing. Workplace conflict, regardless of whether it is task related or relational (e.g., Sonnentag et al. 2013), and bullying at work, negatively impacts both mental and somatic health (Nielsen et al. 2014).

AQ5

\subsection{Traumatic Events}

Some adverse experiences seem to have a particularly long-lasting impact on most individuals (e.g., Lucas et al. 2004; Lucas 2005; 2007). Experiencing traumatic events, like sexual assault, have been associated with severe psychological problems, including posttraumatic stress, self-blame, depression, and adjustment problems (see Campbell et al. 2009, for a review). Women who experience being pressured or forced into having sex by their partner are found to also often have a child with the abusive partner (Messing et al. 2014).

\subsection{Illness, Injury, and Accidents}

Good health is associated with wellbeing and satisfaction (e.g., Diener and Chan 2011; Diener et al. 2017), while illness, becoming injured or involved in accidents have a negative impact (e.g., Lucas 2007; Steptoe et al. 2015). Most people are primarily happy (Diener et al. 2018a), and tend to desire good health over illness, misery, and death. Mothers' health is important not only to the mother, but may also impact her capacity to care for her children and family (e.g., Sitnik et al. 2016; Suarez et al. 2016). An extensive literature exists on the effect of maternal mental illness on risk for psychopathology in her children (e.g., O'Hara and Wisner 2014), particularly maternal depression (e.g., Cuijpers et al. 2015; Goodman 2007). In addition to mental health, illness, injury, or accident involvement may have serious consequences for mothers. For example, beyond the immediate personal effects, she may be hospitalized and thus unable to care for her family and children. Experiencing someone close (family members or friends) becoming ill, injured, or involved in an accident may also directly or indirectly affect mothers' life satisfaction, possibly through added caregiving responsibilities or loss of social support (e.g., Bauer and Sousa-Poza 2015). As a result, both personal illness and injury, or the illness or injury of someone close, may greatly influence the wellbeing of a mother as well as her caregiving abilities. The effect of such health-related stressors is therefore 
important to document and understand.

\subsection{Worries About the Child}

Becoming a mother may be associated with worries about the child, including the child's sleeping patterns, eating habits, health, or general development (e.g., Fisak et al. 2012; Stickler et al. 1991; Walzer 1996). Worry is a subjective response, and may be due to the mothers' own anxiety, rather than to real problems (e.g., disability, illness, behavior problems) with the baby. However, the number of children with chronic illnesses and disabilities has increased linearly over the last decade due at least partly to survival of a growing number of children born pre-term, and with various disorders (Boyle et al. 2011). Prevalence estimates indicate that $15-18 \%$ of children below 18 years have chronic conditions arising in early childhood (Boyle et al. 2011; Halfon et al. 2012). The most common child conditions include emotional, behavioral, and neurological disabilities (Halfon et al. 2012): The children's additional needs for care and support are firmly documented (Boulet et al. 2009). Children suffering from compromised health and disabilities demand enhanced parental attention and supervision (Bernheimer et al. 2003; Coley et al. 2011) that divert attention from other important aspects of family functioning (e.g., other children, parental relationships) and may cause a host of worries and logistical burdens. Any worries a mother may have about her child, whether resulting from the mother's own tendency to worry, or to some actual child developmental concern, may impact her overall wellbeing. To date, little is known about the influence of such stressors compared to other types of stress.

\subsection{Income and Economic Problems}

Economy and income play a very important role in life, providing either freedom of choice, or firm limitations on all aspect of life, from where to live to everyday decisions, acquisitions, and activities. Young couples and forming families may experience many simultaneous strains that may include completing an education, finding and securing a job, finding a place to live (while pregnant), and giving birth to and caring for their very first child. Economic worries are found to negatively affect wellbeing (e.g., Dunn and Norton 2013), and may particularly affect families with small children living in cities where housing and living costs are high and there is substantial 
competition to secure a well-paying job.

In the wellbeing literature, the association between income and wellbeing has generated a famous puzzle - the Easterlin paradox (e.g., Clark et al. 2008; Easterlin 1974, 2013; Easterlin et al. 2010; Graham et al. 2010), referring to the observation that economic growth does not seem to increase average life satisfaction in most wealthy nations. At the same time, income correlates positively with life satisfaction at the individual level in these countries (e.g., Lucas and Schimmack 2009). It is important to note that the effect of income is found to vary with two important factors: (1) how satisfaction is measured and (2) how income is treated in the analyses (Kahneman and Deaton 2010). Log transformed income is found to be positively and linearly associated with cognitive life evaluations (Kahneman and Deaton 2010), while emotional wellbeing (the emotional quality of everyday experiences) shows a curvilinear relationship with income (e.g., Kapteyn et al. 2015). Hence, the average lowincome individual reports both lower life satisfaction and lower emotional wellbeing than the average high-income individual.

\subsection{Societal Effects of Stressors}

As previous research has focused mainly on individuals, little is known about how stressors affect populations, population subgroups, and societies. Relatively rare stressors (e.g., pressured to sexual acts) may explain a minimal amount of variance in the obtained data, yet their impact on those affected may be dramatic. By contrast, relatively common stressors (e.g., relationship dissolution) that yield only small or moderate individual effects, might influence population wellbeing substantially. Information on the prevalence of different stressors in a population and their impact on wellbeing levels, enables estimation of the stressors' effect on the overall population wellbeing. In the current study, we estimated the societal burden of each of the given life stressors on mothers' wellbeing. This burden-on-society index reflects the impact that each stressor has on the overall life-satisfaction of this population. The estimate can be compared to the term "attributable risk" used in epidemiology, signifying the difference in rate of incidence of a condition between an exposed versus an unexposed population. This approach has some resemblance to the 'Global burden of disease' and the notion of disabilityadjusted-life-years (DALY) developed in part by the World Health Organization 
(see Institute for Health Metrics and Evaluation, IHME 2016). However, rather than focusing on disease and health conditions, our approach emphasizes the potentially detrimental effect of various life stressors on the wellbeing of a population.

\section{Rationale and Purpose of the Current Study}

In summary, previous research on life stressors and wellbeing has provided important findings. Yet, current knowledge is limited in several ways, and is largely lacking when it comes to the effect of life stressors on mothers. More specifically, because most studies have focused on one or a few stressors only, there is a need for including a broader set of stressors in order to obtain comparative estimates. Second, as many studies have relied on cross-sectional data, evidence of causal directions is lacking, and longitudinal designs with a potential to examine changes in wellbeing following various stressors are highly warranted. Thirdly, people seem to adapt to adversity, but the time and conditions involved are partly unknown, hence, there is a need for investigations on both short- and long-term effects of various stressors. Fourth, because many studies have been based on small and selected samples with limited power to examine infrequent stressors, large-scale population based studies are needed. Finally, some stressors may have only moderate effects on the affected individuals, yet if they occur frequently, their overall effect in terms of reduced population wellbeing may be substantial. An approach that examine both individual and population level effects is thus warranted.

Building on and expanding our current knowledge of how stressors affect wellbeing, the purpose of this study was to investigate the short and long-term effects of single and multiple life stressors on wellbeing among mothers, measured as overall life satisfaction (LS). Using a large population-based panel of mothers followed for thirty months, we explored the effects of ten life stressors, comprising interpersonal relationships, work, health, and financial problems, on current and future life satisfaction (see Table 1 for a complete list of stressors). In the current study, we first explored the overall effect of life stressors, predicting that the presence of life stressors would negatively affect both short-term and long-term life satisfaction. Secondly, the association between experiencing single versus multiple stressors on short- and long-term satisfaction was explored. Third, we investigated the magnitude of the 
association between individual stressors and short- and long-term satisfaction. Fourth, we explored how individual stressors affected satisfaction over time. We predicted that one of four effect patterns would be found; (1) stressors could create an immediate drop in short-term satisfaction that would wear off over time, (2) stressors could show an immediate negative effect with some improvement over time, or (3) stressors could have a lasting negative impact, or (4) stressors would show a deteriorating effect over time. Lastly, we calculated a "burden on society" score based on the obtained life satisfaction impact of a given stressor and the prevalence of that stressor among our mothers. Using the obtained stressor effect as an indicator of how the stressor might affect the whole family system, the "burden on society" estimate enabled us to explore how individual life stressors have the potential to affect the overall wellbeing of a population.

\section{Table 1}

Life stressors reported at Time 2 and Time 3

\begin{tabular}{|c|c|c|c|c|c|}
\hline \multicolumn{2}{|c|}{ Item } & \multicolumn{2}{|c|}{$\begin{array}{l}\text { T2 } \\
\text { stressors }\end{array}$} & \multicolumn{2}{|c|}{$\begin{array}{l}\text { T3 } \\
\text { stressors }\end{array}$} \\
\hline & & \multirow{2}{*}{$\begin{array}{l}\mathbf{N} \\
9011\end{array}$} & \multirow{2}{*}{$\begin{array}{l}\% \\
19.4\end{array}$} & \multirow{2}{*}{$\begin{array}{l}\mathbf{N} \\
8016\end{array}$} & \multirow{2}{*}{$\begin{array}{l}\% \\
17.3\end{array}$} \\
\hline 1 & $\begin{array}{l}\text { Have you had problems at work or where you } \\
\text { study? }\end{array}$ & & & & \\
\hline 2 & $\begin{array}{l}\text { Have you had problems or conflicts with family, } \\
\text { friends, or neighbors? }\end{array}$ & 8048 & 17.4 & 8268 & 17.8 \\
\hline 3 & Have you had economic problems? & 7546 & 16.3 & 8156 & 17.6 \\
\hline 4 & $\begin{array}{l}\text { Has anyone close to you been seriously ill or } \\
\text { injured? }\end{array}$ & 6973 & 15.0 & 8343 & 18.0 \\
\hline 5 & Have you lost someone close to you? & 4247 & 9.2 & 6208 & 13.4 \\
\hline 6 & Have you been seriously ill or injured? & 1972 & 4.3 & 2266 & 4.9 \\
\hline 7 & $\begin{array}{l}\text { Have you been divorced, separated, or ended } \\
\text { your romantic relationship? }\end{array}$ & 865 & 1.9 & 1633 & 3.5 \\
\hline 8 & $\begin{array}{l}\text { Have you been involved in a serious }\left(\text { car }^{\mathrm{a}}\right) \\
\text { accident, }\left(\text { home }^{\mathrm{a}}\right) \text { fire, or robbery? }\end{array}$ & 453 & 1.0 & 413 & 0.9 \\
\hline 9 & Have you been pressured to sexual acts? & 195 & 0.4 & n.a. & n.a. \\
\hline 10 & $\begin{array}{l}\text { Have you been seriously worried there is } \\
\text { something wrong with your child? }\end{array}$ & 3799 & 8.2 & 3922 & 8.5 \\
\hline
\end{tabular}


$N=46,342$

${ }^{\mathrm{a} O n l y}$ specified in $\mathrm{T} 2$ questionnaire

\section{Methods}

\subsection{Sample and Procedures}

The present study used data from the Norwegian Mother and Child Cohort Study (MoBa), a large prospective population-based study conducted by the Norwegian Institute of Public Health (NIPH, Magnus et al. 2016). The Regional Committee for Medical Research Ethics in Southeastern Norway approved the MoBa Study, and informed consent was obtained from each participant upon recruitment. Data collection started in 1999, and in 2008 the aim of enrolling 100,000 pregnancies was reached, with a total response rate of $40.6 \%$ (Norwegian Mother and Child Cohort Study, Revised Protocol 2010). Questionnaire data were collected three times during pregnancy and at 6, 18, and 36 months postpartum (Q1-Q6). Further rounds of follow-up data have subsequently been collected, but were not used in the current study.

The current study used version VI of the quality-assured MoBa data files. Data were taken from questionnaires Q4 (completed 6 months postpartum; Time 1), Q5 (18 months postpartum; Time 2), and Q6 (36 months postpartum; Time 3), with life satisfaction (LS) reported at T1 and T3, and the experience of life stressors at T2 and T3 (see Fig. 1). As MoBa is an ongoing study, the number of data entries varies across waves (the total sample in Version VI is $N=89,744$ at T1, $N=66,808$ at T2, and $N=51,447$ at T3). ${ }^{1}$ The response rate among participants dropped from $95 \%$ at Q1 to $59 \%$ at Q6 in the NIPH data file version VI. Participants in the present study were selected based on having completed both the T1 and T3 life satisfaction items, resulting in a total sample of 46,342 cases.

\section{Fig. 1}

Data collection points in the current study. Life Satisfaction (LS) was collected at T1 and T3 while data on Major Life Stressors (MLS) was collected at T2 and T3 


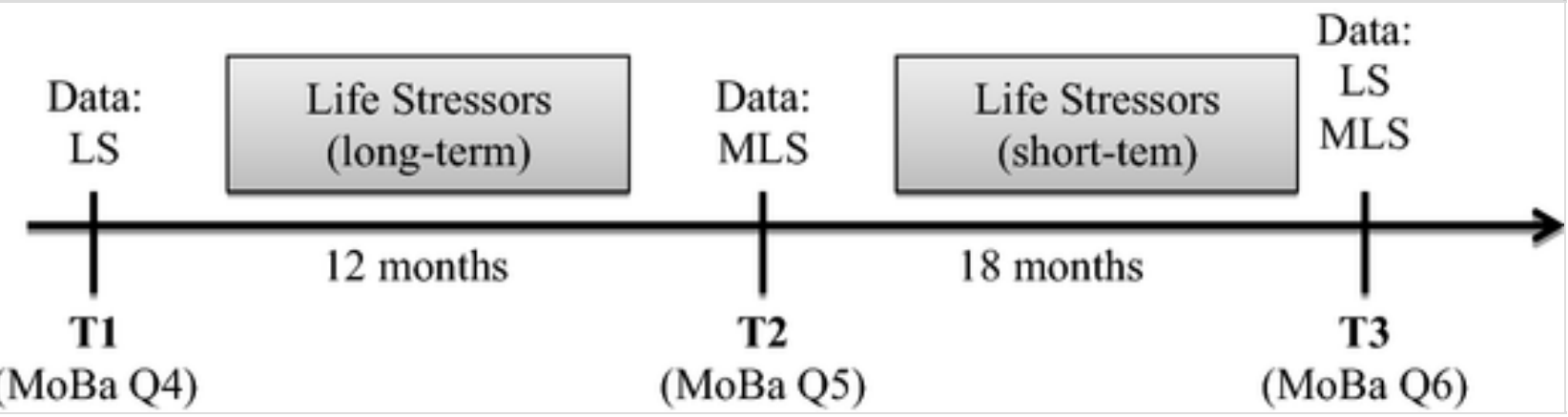

AQ6

\subsubsection{Attrition}

In a previously published study using MoBa data, a survival analysis using Cox regression was run to investigate selective attrition based on initial life satisfaction scores (see Dyrdal et al. 2011, for details). The hazard ratio for dropping out of the study was very close to one (.98), indicating virtually no selective attrition with regard to life satisfaction.

\subsubsection{Sample Characteristics}

The mean age of the sample was 29.9 years $(S D=4.4)$ at T1. Participants in the $\mathrm{MoBa}$ are slightly older, with a higher percentage living in stable relationships than the average Norwegian mother (Nilsen et al. 2009). The relatively low recruitment to the MoBa may produce somewhat biased prevalence estimates. Of note, the exposure outcome associations do not seem to be biased (Nilsen et al. 2009).

\subsection{Measures}

\subsubsection{Life Satisfaction (LS)}

Life satisfaction was measured using The Satisfaction With Life Scale (SWLS) is a widely used instrument for measuring life satisfaction (Diener et al. 1985; Pavot and Diener 2008). This five-item scale shows excellent psychometric characteristics and includes items such as "The conditions of my life are excellent" and "I am satisfied with my life", rated on a 1-7 Likert scale (1 = Strongly Disagree, 7 = Strongly Agree). Individual sum scores (range 5-35) and z-transformed scores were computed and used in the analyses. Cronbach's 
alphas were estimated to be .88 and .91 at Time 1 and 3, respectively.

\subsubsection{Life Stressors}

Data on ten different life stressors were collected at T2 and T3, using a life stressor checklist developed by the MoBa group. The checklist was based on previous work (e.g., Coddington 1972), has been used in recent published studies (e.g., Røsand et al. 2012), and includes recent (last 12/18 months) stressors such as economic problems, conflict with family and friends, relationship dissolution, and experience of illness and death (for a complete list of items, see Table 1). Participants indicated whether they had experienced the given stressor ("yes"/“no") during the past 12 (T2) or 18 (T3) months.

\subsection{Analyses}

To explore the short-term effects of life stressors on life satisfaction reported at T3, we used the life stressor data collected at T3. A short-term effect was defined as the effect of recent life stressors, experienced during the past 18 months (i.e., between T2 and T3) on current LS. To explore long-term effects, we examined the effect of life stressors experienced between $\mathrm{T} 1$ and $\mathrm{T} 2$ (i.e., 18-30 months prior to T3), on life satisfaction reported at T3. To determine whether any observed effect on life satisfaction resulted from the experience of life stressors rather than from some preexisting level of life satisfaction, we also controlled for LS at T1 in some of the analyses.

We chose to run the regression analyses using a dichotomous predictor variable (life stressor: "yes"/"no") and a standardized outcome measure (Z-transformed LS) to obtain the unstandardized regression weights, $B$. This unstandardized regression weight is an effect size, corresponding to Cohen's $d$, and is reported for all sets of analyses. An effect size $<0.2$ is considered small, while effect sizes between .2 and .5 are considered moderate, $0.5-0.8$ medium, and $>0.8$ large (Cohen 1992). All analyses were conducted using IBM SPSS, version 21.0 .

To explore how life stressors affected life satisfaction, we first investigated whether the presence of life stressors in general affected overall life satisfaction. Then we calculated the prevalence of the different life stressors in 
our sample, and explored the impact of experiencing single versus multiple life stressors on short- and long-term LS. To more fully understand the impact of stressors, we further explored short- and long-term effects of life stressors on LS by conducting eight regression analyses. Of these, four explored short-term effects (Analyses I- IV, i), and four explored long-term effects (Analyses I-IV, ii).

\subsubsection{Analyses I}

In Analyses I, individual life stressors were entered as single predictors of standardized LS. Hence, ten multiple regression analyses were run exploring short-term effects, using individual stressors reported at T3 to predict LS reported at T3 (Analyses I-i), and ten analyses exploring long-term effects using individual stressors reported at T2 to predict LS reported at T3 (Analyses I-ii), respectively (displayed in Columns (a) and (e), Table 2).

\section{Table 2}

Unstandardized regression coefficients (B) for the short- and long-term effect o standardized summed life satisfaction (LS) at T3 with control for life satisfaction at T1

\begin{tabular}{|c|c|c|c|c|c|c|}
\hline & \multicolumn{4}{|c|}{ Short-term effects (i) } & \multicolumn{2}{|c|}{ Long -term eff } \\
\hline & Analysis I & II & III & IV & Analysis I & I \\
\hline & $\begin{array}{l}\text { Unadjusted } \\
\text { (a) }\end{array}$ & $\begin{array}{l}\text { Adjusted } \\
\text { (b) }\end{array}$ & $\begin{array}{l}\text { Unadjusted } \\
\text { with LS T1 } \\
\text { (c) }\end{array}$ & $\begin{array}{l}\text { Adjusted } \\
\text { with LS } \\
\text { T1 } \\
\text { (d) }\end{array}$ & $\begin{array}{l}\text { Unadjusted } \\
\text { (e) }\end{array}$ & \\
\hline Life events & $B$ & $B$ & $B$ & $B$ & $B$ & $E$ \\
\hline $\begin{array}{l}\text { Life } \\
\text { satisfaction } \\
\text { T1 }\end{array}$ & & & & $.52 * *$ & & \\
\hline $\begin{array}{l}\text { Problems at } \\
\text { work }\end{array}$ & $-.36^{* *}$ & $-.22 * *$ & $-.23 * *$ & $-.16^{* *}$ & $-.21 * *$ & - \\
\hline Conflict & $-.48^{* *}$ & $-.29 * *$ & $-.28 * *$ & $-.18^{* *}$ & $-.42 * *$ & - \\
\hline $\begin{array}{l}\text { Economic } \\
\text { problems }\end{array}$ & $-.77 * *$ & $-.59^{* *}$ & $-.46^{* *}$ & $-.36^{* *}$ & $-.62 * *$ & - \\
\hline $\begin{array}{l}\text { Close } \\
\text { seriously ill }\end{array}$ & $-.17^{* *}$ & $-.05 * *$ & $-.11^{* *}$ & $-.04 * *$ & $-.13 * *$ & - \\
\hline
\end{tabular}




\begin{tabular}{|c|c|c|c|c|c|}
\hline $\begin{array}{l}\text { Someone } \\
\text { dying }\end{array}$ & $-.10 * *$ & -.01 & $-.07 * *$ & -.02 & $-.06 * *$ \\
\hline $\begin{array}{l}\text { Worry about } \\
\text { child }\end{array}$ & $-.37 * *$ & $-.18^{* *}$ & $-.20 * *$ & $-.09 * *$ & $-.27 * *$ \\
\hline Seriously ill & $-.48^{* *}$ & $-.27 * *$ & $-.31 * *$ & $-.20 * *$ & $-.45^{* *}$ \\
\hline $\begin{array}{l}\text { Relationship } \\
\text { dissolution }\end{array}$ & $-1.15^{* *}$ & $-.85 * *$ & $-.76^{* *}$ & $-.61 * *$ & $-.93 * *$ \\
\hline Accident & $-.24 * *$ & .04 & $-.09 *$ & $.10^{*}$ & $-.19 * *$ \\
\hline $\begin{array}{l}\text { Pressured } \\
\text { to sexual } \\
\text { acts }\end{array}$ & & & & & $-1.05^{* *}$ \\
\hline Constant & & $.27 * *$ & & $.18^{* *}$ & \\
\hline
\end{tabular}

$* * p<.001 ; * p<.01$

$\mathrm{B}=$ Unstandardized regression weight (corresponding to Cohen's $d$ ); Adjusted $=$ reg other stressors. With LS T1 = analyses include a control for Time 1 Life Satisfactior of analyses described in the analysis section

\subsubsection{Analysis II}

In Analyses II, regression analyses were run using all ten life stressors as simultaneous predictors of short- (i) and long-term (ii) LS (see Columns (b) and (f) in Table 2, respectively).

\subsubsection{Analyses III}

Analyses III repeated the multiple regressions conducted in Analyses I, adding a control for previous levels of satisfaction (entering LS reported at T1 and each individual stressor as predictors of LS at T3). This LS T1 control was added when exploring both short-term (i) and long-term (ii) effects (see Column (c) and $(\mathrm{g})$, Table 2, respectively).

\subsubsection{Analysis IV}

In Analysis IV, we repeated Analysis II, adding a control for prior (T1) life satisfaction levels (i.e., entering all ten life stressors as simultaneous predictors of short- (i) and long-term (ii) life satisfaction, in addition to LS at T1). 


\section{Results}

Participants reported high levels of life satisfaction at both T1 and T3, with a mean LS sum score of $28.97(S D=4.75)$ and $27.49(S D=5.44)$ at $\mathrm{T} 1$ and T3, respectively. Life satisfaction decreased slightly between $\mathrm{T} 1$ and $\mathrm{T} 3$. This reduction is assumed to reflect a natural decrease in LS following the birth of a child (see Dyrdal et al. 2011).

The most commonly experienced life stressor was problems at work, reported by $19.4 \%$ of the sample. This was followed by experiencing someone close becoming ill or injured (18.0\%), conflict with family, friends, or neighbors (17.8\%), and economic problems (17.6\%). Being pressured to sexual acts was included as a stressor only at T2 and was a rare event, reported by only $0.4 \%$ of the participants in the total sample (see Table 1 for a full list of stressors and frequencies at $\mathrm{T} 2$ and $\mathrm{T} 3$ ).

\subsection{The Effect of Multiple Stressors}

Of the total sample, $52.3 \%$ (24,216 women) reported having experienced one or more life stressors between $\mathrm{T} 1$ and $\mathrm{T} 2$, while $54.6 \%$ (25,284 women) experienced one or more stressors between T2 and T3 (see Table 1 and Fig. 2). The association between LS and number of life stressors was negative and linear. Figure 2 shows sum-score LS levels according to the number of stressors reported. Individuals who did not report life stressors had a short-term summed LS score of 29.04 and a long-term LS score of 28.78. Regression analyses (with standardized LS as outcome) yielded small to moderate effect sizes for both short-term $(B=-.26, t(44,42)=-67.58, p<.001)$, and long-term $(B=-.22, t$ $(41,42)=-52.82, p<.001)$, effects. Thus, for each additional stressor experienced, we estimated a reduction in short- and long-term LS of approximately $0.25 S D$. Translating this effect into reduced life satisfaction implies that participants experiencing four life stressors during the past 18 30 months would report a one standard deviation lower LS compared to respondents not reporting any stressors.

\section{Fig. 2}

Overall summed life satisfaction among participants experiencing multiple life stressors. Note Total $N=46,342$. Short term 0 stressors: $N=19,140 ; 1$ stressor: $N$ 
$=13,032 ; 2$ stressors: $N=7385 ; 3$ stressors: $N=3175 ; 4$ stressors: $N=1206,5$ stressors: $N=351 ; 6$ stressors: $N=107$. Long term 0 stressors: $N=17,212 ; 1$ stressor: $N=12,760 ; 2$ stressors: $N=7104 ; 3$ stressors: $N=2949 ; 4$ stressors: $N=$ 1029, 5 stressors: $N=288$; 6 stressors: $N=63$

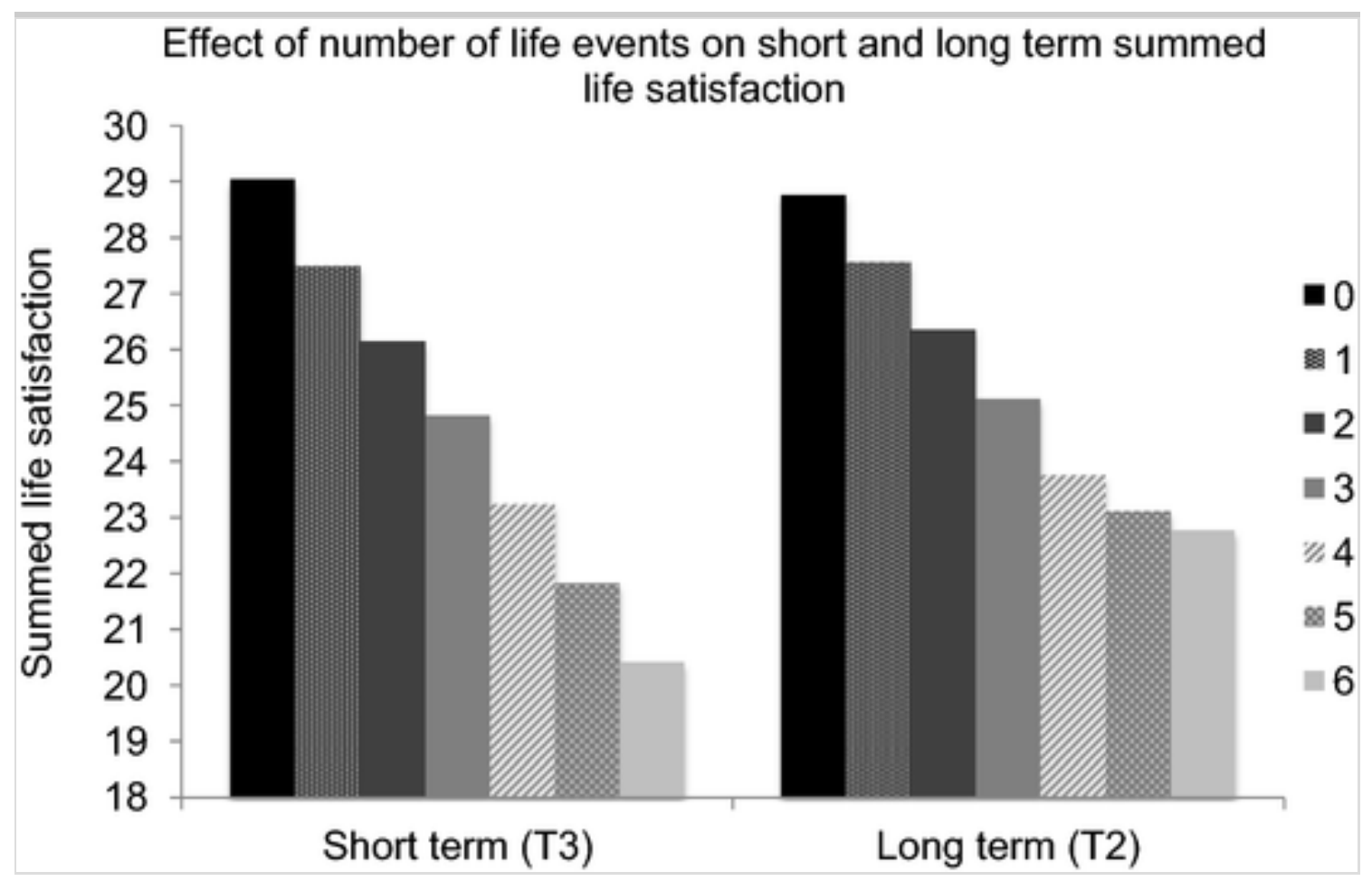

\subsection{Short-Term Effects of LIFE should be lower-case letters; Life Stressors on Life Satisfaction}

Four regression analyses were run exploring the association between current life satisfaction and life stressors experienced during the past 18 months (using life stressor and satisfaction data collected at T3).

\subsubsection{Analyses I}

In Analyses I (Column (a), Table 2), individual stressors were regressed on short-term standardized LS. Results revealed a significant and negative association between all life stressors and LS. Relationship dissolution was associated with a reduction in life satisfaction of more than one standard deviation $(B=-1.15, p<.001)$. Also, economic problems, being seriously ill, or conflict with family or friends were associated with substantially lower LS scores. The effect sizes of these three stressors were moderate to medium, with 
an associated reduction in standardized LS by a half to $3 / 4$ of a standard deviation.

\subsubsection{Analysis II}

To investigate the unique contribution of each life stressor on short-term satisfaction, we entered all stressors simultaneously as predictors of LS in Analysis II (see Column (b), Table 2). Relationship dissolution emerged as the strongest negative predictor of LS $(B=-0.85, p<.001)$, followed by economic problems and conflict with family or friends, all with moderate to medium effect sizes (a reduction in LS of between .29 and .85 standard deviations). Experiencing someone close dying or being involved in an accident were not significant predictors.

\subsubsection{Analyses III}

In Analyses III (Column (c), Table 2), we repeated Analysis I displayed in Column (a), controlling for prior levels of life satisfaction to enable an examination of the association between life stressors and subsequent change in life satisfaction. Hence, life satisfaction scores obtained at T1 (i.e., 6 months postpartum), and life stressor data at T3 (i.e., collected at 36 months postpartum) were entered into the analyses to predict T3 life satisfaction (36 months postpartum). Results showed that even after controlling for prior levels of satisfaction, all stressors had significant negative short-term effects on satisfaction. The strongest effects emerged for relationship dissolution and economic problems, being associated with a reduction in short-term life satisfaction of $B=.76, p<.001$ and $B=.46, p<.001$, respectively. Effects sizes for the remaining stressors were small to medium.

\subsubsection{Analysis IV}

In Analysis IV (Column (d), Table 2), all life stressors and T1 LS were entered to explore the unique impact of individual stressors on LS change across time. All stressors except experiencing someone close dying significantly predicted changes in LS, yielding small to medium effect sizes. Relationship dissolution and economic problems were the strongest predictors, followed by being seriously ill or injured and conflict with family or friends. 


\subsection{Long-Term Effects of Life Stressors on Life Satisfaction}

To investigate long-term effects of life stressors, we reran Analyses I-IV described above using life stressors reported at T2 (i.e., stressors experienced between T1 and T2) as predictors of life satisfaction at T3. Hence, these life stressors were experienced 18-30 months prior to reporting LS. The results are displayed in Columns (e) to (f) in Table 2.

\subsubsection{Analyses I}

In Analyses I [Column (e)] Use regular parenthesis ,, life stressors were entered individually as predictors of long-term LS in a series of multiple regression analyses. All of the examined life stressors were associated with a significant reduction in life satisfaction, with effect sizes ranging from small to large (Cohen 1992). The largest negative effects were found for being pressured to sexual acts and for relationship dissolution. These life stressors were associated with a reduction in life satisfaction of close to one standard deviation.

\subsubsection{Analysis II}

In Analysis II, all stressors were entered simultaneously as predictors of T3 life satisfaction. Being pressured to sexual acts, relationship dissolution, and economic problems all had moderately strong negative long-term effects, while no long-term effects were indicated for someone close dying or being involved in an accident or seriously injured (see Column (f) for details).

\subsubsection{Analyses III}

When controlling for previous levels of life satisfaction in Analyses III, all life stressors showed a significant and long-term negative effect on satisfaction, with being pressured to sexual acts, relationship dissolution, and economic problems showing the strongest effects. These stressors reduced long-term life satisfaction with .30 to .45 standard deviations [see Column $(\mathrm{g})$ ].

\subsubsection{Analysis IV}

Entering all stressors and prior life satisfaction as predictors in Analysis IV, being pressured to sexual acts, economic problems and relationship dissolution 
emerged as moderately strong negative predictors of long-term satisfaction [see Column (h)] Regular parenthesis . Experiencing someone close dying or being seriously ill or injured did not predict long-term life satisfaction.

\subsection{Short and Long-Term Effects of Individual Stressors: Effect Patterns}

To explore the magnitude of each unique stressor's effect on short- and longterm summed life satisfaction, we re-ran the analyses shown in Table 2, Columns (b) and (f), using the life satisfaction sum score value (5-35 scale). Taking the variance differences at T2 and T3 into account, we obtained $B$ coefficients. To obtain stressor effect patterns, we subtracted the unique effect of each stressor (the $B$-coefficient) from the mean satisfaction level of participants not reporting any stressors (i.e., using the summed life satisfaction score of participants not reporting any stressors as a baseline/zero). The resulting life satisfaction trajectories are shown in Fig. 3. All life stressors, apart from being involved in an accident, negatively affected LS. However, individual stressors affected short- and long-term satisfaction differently. Relationship dissolution strongly reduced short-term LS (by 3.6 summed satisfaction points), and although a slight improvement in life satisfaction was found over time, it remained below baseline throughout the study, with mothers experiencing relationship dissolution reporting a long-term reduction in life satisfaction of 1.3 summed LS points. Similar effect patterns were observed for economic problems and problems at work, showing larger short-term than long-term effects, thus indicating some improvement over time. Surprisingly, being involved in an accident had a positive short-term and no long-term effect on life satisfaction. This might result from downward counterfactual thoughts about how the situation could have turned out worse than it actually did (e.g., Teigen and Glad 2011). Experiencing someone close becoming ill and worrying about one's child had a lasting negative effect, with no improvement shown over time.

\section{Fig. 3}

Short-term (past 18 months) and long-term (past 18-30 months) effects of life stressors on summed life satisfaction when controlling for all other stressors. Baseline (zero) represents the mean LS level among respondents not affected by any stressors 


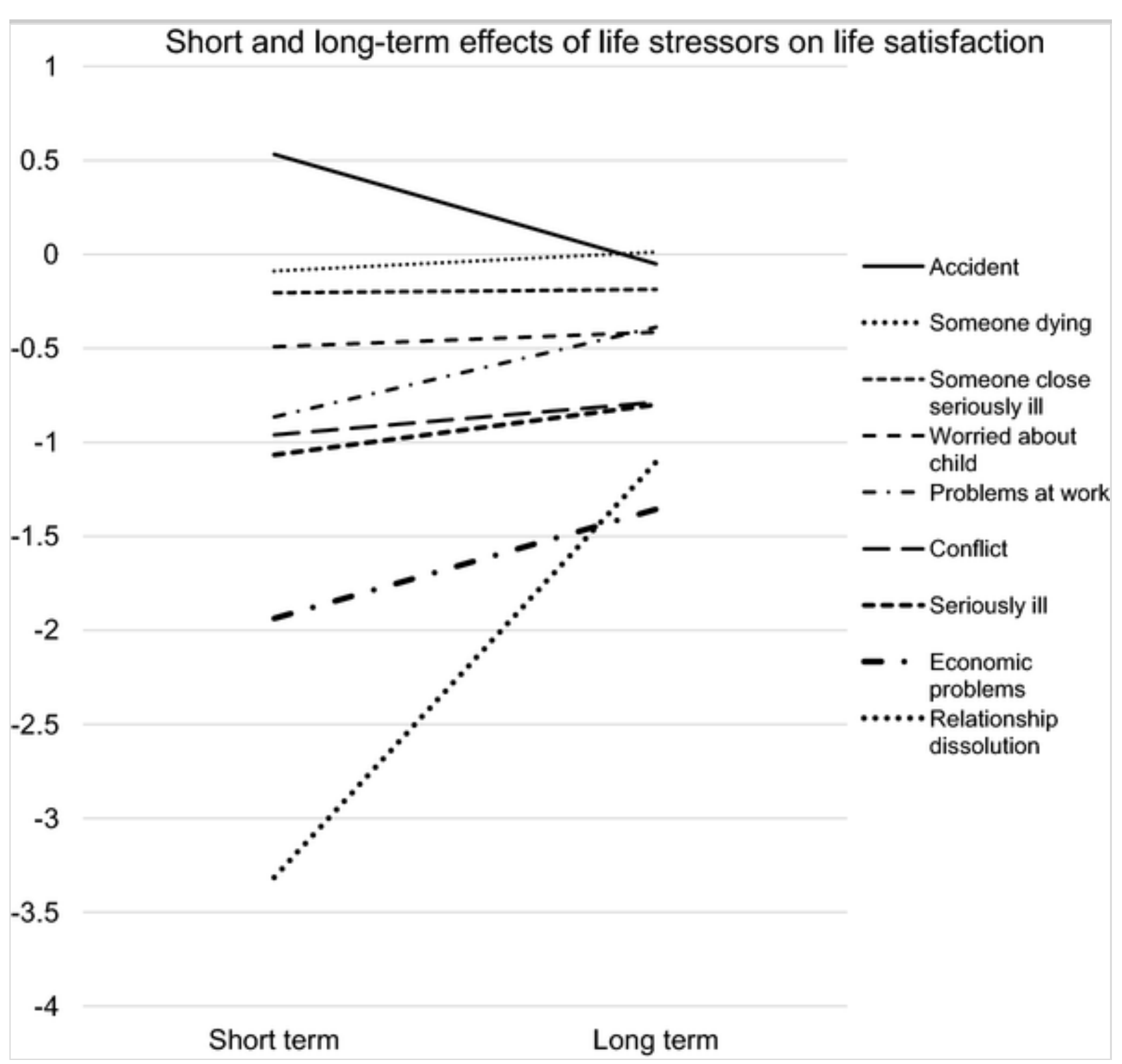

\subsection{Burden on Society}

Mental health problems and diminished life satisfaction are linked to poorer individual, familial, and societal outcomes, and even mild levels of distress might have detrimental effects on individuals and their families. In order to examine the effect of different life stressors on overall societal life satisfaction levels, we computed a "burden on society" index using summed life satisfaction scores (scale 5-35). To compute this index, we used the unique effect of each life stressor obtained from the adjusted regression analyses, controlling for prior levels of satisfaction. The analysis is similar to the ones displayed in Columns (d) and (h), Table 2, but were run with summed LS as the dependent variable. We calculated the burden on society index as the product of the obtained effect 
sizes (i.e. unstandardized regression weight, $B$ ) and the proportion of the sample exposed to the stressor, $n$ ( see "Appendix" Change to "see Table 3 in the Appendix for details" for details). For example, calculating the burden on society for recent relationship dissolution (short-term effect), we multiplied the obtained regression weight for summed life satisfaction $(B=-3.31)$ by the frequency of occurrence in the current sample $(3.5 \%=0.035)$, equaling -0.116 . To make this value more interpretable, the effect of relationship dissolution in a population of 100 people is found by multiplying the obtained number $(-0.116)$ with the population size, in this case by 100 . The resulting number, -11.60 , reflects the total number of summed life satisfaction points relationship dissolution deducts from the overall summed satisfaction of a population of 100 individuals. Individuals not experiencing any stressors were found to have a summed satisfaction of 29.04. Hence, in a population of 100 individuals, relationship dissolution will reduce the overall short-term satisfaction from 2904 to 2892.4. Calculating the overall burden on society for all stressors, economic problems, conflict with family and friends, and problems at work were found to have substantial short- and long-term negative impact at the societal level (Fig. 3). For example, in a group of 100 people, perceived economic problems will reduce their total short-term summed life satisfaction 34 points $((1.9 \times 0.176) \times 100=34.1)$, or correspondingly, deduct more than 340,000 life satisfaction points from a population of one million people.

\section{Discussion}

The purpose of this study was to explore short- and long-term effects of different life stressors on the life satisfaction of mothers. Most studies within the realm of wellbeing have had a tendency to adopt a positive focus, exploring positive outcomes (like resilience, positive emotions, optimism), using positive predictors (e.g., social support, feelings of mastery) (see Diener et al. 2018b, for a review). Overall, people are found to have a general tendency to be happy (Diener and Diener 1996; Diener et al. 2018a, b). Yet, certain conditions and life circumstances are associated with reduced wellbeing (e.g., Diener and Diener 2017) Should be "Diener et al., 2018" .. Stressors and conditions that contribute to reduced wellbeing can be thought of as "thieves of happiness", and the present study explored the effect of life stressors in this context. Because participants were followed for 3 years, an exploration of the impact of different stressors (both in duration and absolute effects) was possible. The phase 
following childbirth is critical in several ways. New dynamics may develop as a new person enters the family, and relationships and attachment patterns within the family may change as parents and siblings adjust. To ensure the development of healthy families, with good and strong parental bonds, healthy attachments among children and parents, and sustainable communication patterns, reducing unnecessary stressors may be of particular importance. Going through pregnancy, birth, and the postpartum period requires that mothers have enough resources to handle these changes in a good way, and added external stressors may overload her coping resources. Exploring how external stressors may influence mothers in these forming years is essential to secure family health and healthy development. Identifying stressors that may be particularly negative in this phase can enable policymakers to prioritize and develop interventions or programs, or provide social benefits that will benefit individuals or families in need. The current study provides important information on these issues, using data from a large panel of mothers followed for 3 years. The effect of life stressors on short- and long-term maternal life satisfaction was investigated. As previous studies have mainly explored the impact of single life stressors rather than compared the effect of different stressors or events within a single study, the present study was able to remedy this by comparing the effect of ten different life stressors on life satisfaction. Additionally, we set out to explore the absolute and relative effects of these stressors on short and long-term life satisfaction, as well as their impact on overall, societal wellbeing.

As predicted, we found that life stressors significantly reduced participants' life satisfaction, with a linear and negative association between number of stressors reported and life satisfaction. For each additional stressor reported, a corresponding drop of .26 standard deviations in summed satisfaction was found. Not only recent stressors, but also stressors occurring 18-30 months prior to data collection, were associated with reduced life satisfaction (see Fig. 2). Using data collected at T3, participants reporting no major recent life stressors had an average summed life satisfaction score of 29.0, while participants experiencing six stressors over the past eighteen months reported an average life satisfaction score of 20.4 .

\subsection{Short- and Long-Term Individual Stressors}


Comparing the effects of individual stressors (by controlling for the effect of the other stressors) resulted in an overall reduction of effects. Relationship dissolution emerged as the strongest predictor of short-term satisfaction, corroborating previous research on the negative association between life satisfaction and divorce (e.g., Lucas 2005; Lucas and Clark 2006; Næss et al. 2015; Oishi 2012). Divorce not only affects parents, as research is showing increased symptom load for children living in joint physical custody where they spend considerable time in the two different households after a parental divorce (e.g., Bergström et al. (2014). Economic problems and being seriously ill had moderate to medium short-term effects, while being involved in an accident and experiencing someone close dying had no significant unique effects. Although this latter finding might seem somewhat surprising, other studies have reported similar results (e.g., Marum et al. 2013). Accidents may result in different degrees of injury, some demanding long recovery times and considerable life adjustments, others hardly affecting the person at all. As such, the effect of experiencing an accident may vary greatly, even producing relief and a feeling of being lucky for an outcome that could have turned out much worse (e.g., Teigen and Glad 2011). Experiencing someone close dying might also produce a variety of reactions, depending on the nature of the death. An expected death, like that of an old or sick relative, can produce a very different reaction than a death resulting from a sudden and unexpected event, like a car accident. In the face of these events, emotional reactions are natural, normal, and expected. However, these reactions may not be captured well enough by the life satisfaction scale used in the present study. Rather, such emotional reactions are found to be better captured using clinical scales, or scales measuring emotional reactions (e.g., sadness, sorrow, worry) more specifically (e.g., Nes et al. 2014b).

Daily conditions that linger over time, or the low-intensity irritations of daily life, are assumed to affect us differently than more final, discrete life events. Such daily hassles have been defined as "experiences and conditions of daily living that have been appraised as salient and harmful or threatening to the endorser's well-being" (Lazarus 1986, p. 40). Previous research has suggested that daily hassles are better predictors of wellbeing and life satisfaction than discrete life events (e.g., Chamberlain and Zika 1990). Economic problems and illness can be viewed as such lingering conditions, resembling daily hassles, 
while the death of a close friend or relative constitutes a discrete and final event. This distinction and the effect of discrete and final "life event" versus the more enduring, often low-intensity hassles on life satisfaction is worth exploring further in future studies.

In the current study, all life stressors were associated with a long-term reduction in life satisfaction. The largest reductions in long-term satisfaction were found for being pressured to sexual acts, relationship dissolution and economic problems. The strong and enduring negative effect of being pressured to sexual acts supports previous research indicating that sexual abuse may have a particularly devastating and long-lasting impact on those affected (e.g., Campbell et al. 2009). When controlling for other stressors, relationship dissolution yielded strong negative effects, supporting previous findings demonstrating that good interpersonal relationships constitutes a major source of happiness for most people (Diener and Seligman 2002). Experiencing problems in other close relationships beyond the marital relationship was also expected to have a strong negative impact on life satisfaction, and our results showed that the stressor conflict with family or friends was significantly, but more moderately, associated with a long-term reduction in life satisfaction than relationship dissolution. Interpersonal conflict thus seems to constitute a central source of long-term dissatisfaction.

Controlling for the effect of other stressors, the current study also found a negative short- and long-term effect of economic problems and problems at work on life satisfaction. Economic problems may result from unemployment, and unemployment is commonly shown to create lasting reductions in wellbeing (e.g., Lucas et al. 2004). The economic uncertainty following unemployment is associated with development of anxiety, depression (Pearlin et al. 1981), and increased mental problems (Goldman-Mellor et al. 2010). Unemployed young adults are found to experience more mental health problems than those having a job (Reneflot and Evensen 2014). Employability, the ability of an individual to find and sustain employment, has also been found to moderate the effect of unemployment on life satisfaction (Green 2011).

Becoming seriously ill or injured yielded a small long-term reduction in life satisfaction. This is in accordance with past research, showing that disability might lastingly alter life satisfaction levels (e.g., Lucas 2007). 
According to the results of past research, life events such as marriage, disability, and divorce may affect life satisfaction differently over time (Lucas 2005, 2007; Lucas and Clark 2006; Luhmann et al. 2011), and an additional aim of the current study was to investigate this. Economic problems and problems at work were associated with a drop in life satisfaction, followed by an improvement over time (see Fig. 3). By contrast, worrying about one's child and having someone close becoming seriously ill showed a lasting negative impact on satisfaction levels. This is in line with studies reporting long-lasting psychological distress and compromised life satisfaction following the birth of a chronically disabled child (Nes et al. 2014b). Extensive childhood care needs are strongly associated with impaired mental health in mothers during early motherhood, with excess risk of a long-term sick leave due to psychiatric disorders more generally and depression more specifically (Hauge et al. 2015). However, in the current study, the question is whether the mother worried about the child, not if the child had impaired health. Worrying is a subjective response, and as such, the question may signify a subjective condition in the mother rather than a childhood condition or disability. This complicates interpretation of findings with regard to this particular life stressor.

Being involved in an accident showed a positive short-term effect, dropping just below baseline over time. This is in accordance with studies finding that positive short-term effects of life stressors are not uncommon and might reflect processes like "benefit finding", "post-traumatic growth" (e.g., Taylor 2012), relief (Teigen and Glad 2011), and adaptation more generally. Accidents might for example cause people to pause and appreciate their life more, experiencing relief that they were not more seriously injured, and consequently yielding greater satisfaction with life. The elevated satisfaction levels observed following experiencing an accident in this study did not last, however, and 18 months following the accident or injury, life satisfaction levels were no longer affected.

None of the life stressors included in the current study showed increased negative effects over time, rather, their negative effects decreased. So even though life stressors may act as "thieves of happiness", over time humans seem to have a natural tendency and ability to adjust and adapt to most situations. Yet, different stressors in the current study did affect satisfaction levels to 
different degrees, with some being more long-lasting than others. This is important knowledge, as mothers' functioning plays a particularly important role for her developing child in the years following birth. Knowing the effect of different stressors on mothers and the family system will enable policy makers and clinicians to make informed decisions when prioritizing efforts or interventions that can help sustain or enhance the wellbeing of mothers and families.

\subsection{Burden on Society}

While life stressors were found to negatively affect the life satisfaction of individuals, an important remaining question concerns the extent to which life stressors affect the life satisfaction of a population, or societal life satisfaction levels. Common stressors, even those having only a modest effect on individual satisfaction levels, might impact societal satisfaction levels quite substantially. Similarly, relatively rare life stressors having a large negative impact on the individual may exert a relatively small effect on societal satisfaction levels. As most societies aim to be happy and thriving, preventing highly stressful life events and making efforts to counteract the adverse effects of common life stressors, including those with small to moderate individual effects, may be crucial. Family economic problems are related to delay or dysfunction in most domains of children's development (Dearing 2014), but the adverse effects may be buffered by both individual and public measures. A Norwegian study has for example shown that high quality Early Childhood Education and Care (ECEC) may buffer children (e.g., internalizing problems) from the negative effects of family income dynamics (Zachrisson and Dearing 2015).

In the current study, we created a burden on society index to investigate the effects of life stressors on the overall life satisfaction of our population. This "burden on society index" was calculated by multiplying the individual stressor's overall impact (i.e., the unstandardized regression weight from the adjusted regression analyses) with the prevalence of the stressor in the population (i.e., the frequency of occurrence of the particular stressor in the present sample). The resulting value indicated the overall reduction in summed life satisfaction created by the stressor per 100 people. This index can be seen as a parallel to, and as complementing, the "Global Burden of Disease" (Murray and Lopez 1997) index. However, rather than quantifying health loss due to 
illnesses and diseases, the "burden on society" index quantifies health loss due to life stressors or events. Many life stressors may be fully or partly preventable, hence, this index may prove important for policy makers in prioritizing health promoting efforts and interventions. Knowing the overall health effects of stressors in different populations, different regions, as well as in different countries, can be important for both prevention and treatment, on the international, national, as well as the regional and municipal level. This index may also prove important as a tool for identifying vulnerable groups or populations (e.g., those exposed to multiple stressors simultaneously), when prioritizing social benefits or designing targeted interventions. In the current study, the societal burden of economic problems, conflict and problems at work was found to be substantial (see Fig. 4). These three stressors reduced shortterm overall life satisfaction of 100 mothers by between 15 and 34 points, while long-term satisfaction was reduced by between 8 and 22 points. This implies that a population of one million people will suffer a loss in short-term wellbeing of between 150,000 and 340,000 life satisfaction points. The long-term loss will be between 80,000 and 220,000 life satisfaction points. Coupled with findings showing that happy people have strong social relationships and are more positive (Diener and Seligman 2002), have better health (Howell et al. 2007), and are more satisfied with their jobs and perform better (Boehm and Lyubomirsky 2008), individuals and societies would benefit considerably from limiting such stressors and their effects. Using the "burden on society" index, further research may help identify what stressors are the biggest "thieves of wellbeing", identifying vulnerable groups or populations, comparing efforts across countries, and designing societal interventions or programs for those at elevated risk.

\section{Fig. 4}

The societal effect of experiencing each life stressor, controlled for the effect of other stressors. The bars denote the amount of life satisfaction points (on a summed scale) each stressor subtracts from the overall life satisfaction of 100 people 


\section{Burden on society}

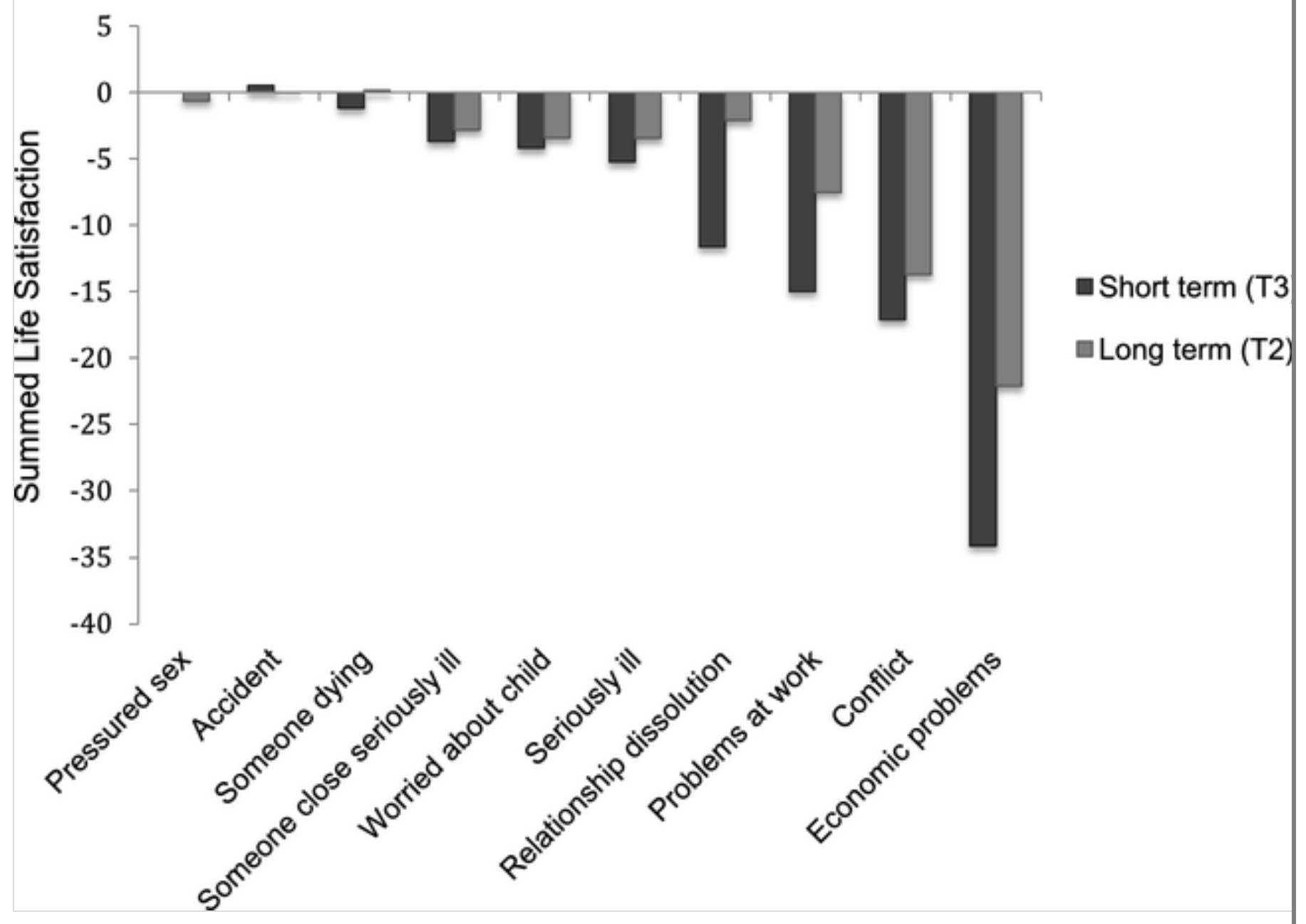

\section{Strengths, Limitations, and Directions for Future Studies}

Use of longitudinal data has numerous advantages, including the ability to investigate temporal effects and changes across time. As most previous studies have explored correlates of wellbeing, a unique characteristic of the present study is that its design enabled us to explore possible causal effects.

Furthermore, large population-based studies like the present one enable an investigation of rare stressors and their effects on wellbeing, and the $\mathrm{MoBa}$ is also strengthened by its inclusion of standardized questionnaires showing excellent psychometric properties (like the SWLS; Diener et al. 1985). Also, the opportunity to directly compare the effect of different stressors, and to explore the effect of multiple concurrent stressors on satisfaction, is a major advantage of the current study. Having a baby may in itself be considered a life stressor. In this study, however, childbirth is not examined as a stressor, as it has been 
explored elsewhere (see Dyrdal et al. 2012 Should be 2011 ). Our ability to study a cohort of women during early motherhood might also constitute a strength of the current study. Previous research has focused on the transition to parenthood and the stresses associated with becoming parents, on how the parental relationship might suffer in this period of life, on maternal health as well as on postpartum depression (see Roy et al. 2014). However, less is known about the effects of more naturally occurring life stresses on mothers during this phase. As such, the current study is an important contribution in exploring different thieves of wellbeing and how to uphold maternal health through this life phase, as knowledge on how stressors affect women in this particular phase is highly warranted and largely lacking. Secondly, participants in the current study were followed for 30 months. Although this is a relatively long follow-up period, future studies are encouraged to include even longer follow-up periods to more extensively explore the duration of effects. Third, while the present study primarily investigated the effect of time-limited stressors, effort should be made in future studies to explore the effect of more lasting stressors on satisfaction. Furthermore, daily hassles have been found to better predict wellbeing than more discrete life events (e.g., Chamberlain and Zika 1990), and consequently, low frequency daily irritations should be included as stressors in future studies. Lastly, given the nature of the current study, individuals from ethnic minorities and lower socioeconomic groups are underrepresented in the current sample. A possible effect is that the frequencies of reported stressors in the current sample might be lower than would be expected given a more representative sample. Hence, our findings on the societal burden of stressors are likely to represent under-estimates of true population values. To remedy this, future studies should make an extra effort to recruit participants from these underrepresented groups.

Despite a number of advantages, the nature of the current study also provides some limitations. First, all participants were relatively young women from a country with high average life satisfaction. Thus, the results may not generalize to other population subgroups or mothers in less advantaged countries. Future studies should also make an effort to include both men and women of different ages and nationalities to explore gender, age, and cultural effects.

\section{Summary}


The present study found that life stressors negatively affected both short-term and long-term life satisfaction. However, different stressors impacted life satisfaction levels differentially - in terms of both degree and duration. Experiencing multiple stressors reduced satisfaction in a linear manner, with each stressor resulting in a 0.25 standard deviation reduction. The stressors relationship dissolution, economic problems, and being pressured to sexual acts had medium to large negative effects on both short- and long-term life satisfaction, while the death of someone close had an immediate negative effect disappearing over time. Individuals being involved in an accident had a higher short-term life satisfaction than individuals not reporting life stressors, but this effect disappeared over time.

While being pressured to sexual acts and experiencing relationship dissolution strongly affected life satisfaction levels, the 1-year prevalence was relatively low in this sample, implicating modest effects at the societal level. By contrast, economic problems, problems at work, and being in conflict with family or friends, were reported by a substantial number of participants. Thus, despite their unique individual level impact being more modest, these life stressors do constitute a substantial overall burden on societal life satisfaction.

\section{Acknowledgements}

The Norwegian Mother and Child Cohort Study is supported by the Norwegian Ministry of Health and the Ministry of Education and Research, NIH/NIEHS (Contract No NO-ES-75558), NIH/NINDS (Grant No. 1 UO1 NS 047537-01), and the Norwegian Research Council/FUGE (Grant No. 151918/S10). We are grateful to all participating families in Norway who take part in this ongoing cohort study.

\section{Appendix}

\section{See Table 3. Delete}

\section{Table 3}

Values used to calculate the Burden on Society Index 


\begin{tabular}{|c|c|c|c|c|c|c|}
\hline & \multicolumn{3}{|c|}{ Short term } & \multicolumn{3}{|c|}{ Long term } \\
\hline & $\begin{array}{l}\text { Summed } \\
\text { LS }\end{array}$ & $\%$ & Burden & $\begin{array}{l}\text { Summed } \\
\text { LS }\end{array}$ & $\%$ & Burden \\
\hline Someone dying & -0.09 & 13.4 & -1.19 & 0.01 & 9.2 & 0.12 \\
\hline Accident & 0.53 & 0.9 & 0.48 & -0.05 & 1 & -0.05 \\
\hline $\begin{array}{l}\text { Pressured to sexual } \\
\text { acts }\end{array}$ & & & & -1.62 & 0.4 & -0.65 \\
\hline $\begin{array}{l}\text { Someone close } \\
\text { seriously ill }\end{array}$ & -0.20 & 18 & -3.67 & -0.19 & 15 & -2.79 \\
\hline Seriously ill & -1.07 & 4.9 & -5.23 & -0.80 & 4.3 & -3.44 \\
\hline $\begin{array}{l}\text { Relationship } \\
\text { dissolution }\end{array}$ & -3.31 & 3.5 & -11.60 & -1.10 & 1.9 & -2.10 \\
\hline Problems at work & -0.86 & 17.3 & -14.96 & -0.39 & 19.4 & -7.51 \\
\hline Conflict & -0.96 & 17.8 & -17.09 & -0.79 & 17.4 & -13.69 \\
\hline Economic problems & -1.94 & 17.6 & -34.09 & -1.36 & 16.3 & -22.11 \\
\hline Worried about child & -0.49 & 8.5 & -4.18 & -0.41 & 8.2 & -3.39 \\
\hline
\end{tabular}

\section{References}

Ahrens, C. J. C., \& Ryff, C. D. (2006). Multiple roles and well-being:

Sociodemographic and psychological moderators. Sex Roles, 55, 801-815. https://doi.org/10.1007/s11199-006-9134-8.

Ainsworth, M. S. (1991). Attachments and other affectional bonds across the life cycle. In C. M. Parkes, J. Stevenson-Hinde, \& P. Marris (Eds.), Attachment across the life cycle. London: Tavistock/Routledge.

Barimani, M., Vikström, A., Rosander, M., \& Berlin, A. (2017). Facilitating and inhibiting factors in transition to parenthood-Ways in which health professionals can support parents. Scandinavian Journal of Caring Sciences. https://doi.org/10.1111/scs.12367. 
Barnett, R. C., \& Hyde, J. S. (2001). Women, men, work and family: An expansionist theory. American Psychologist, 56, 781-796.

Bauer, J. M., \& Sousa-Poza, A. (2015). Impact of informal caregiving on caregiver employment, health, and family. Journal of Population Ageing, 8, 113-145. https://doi.org/10.1007/s12062-015-9116-0.

Ben-Zur, H. (2012). Loneliness, optimism, and well-being among married, divorced, and widowed individuals. The Journal of Psychology: Interdisciplinary and Applied, 146, 23-36.

Bergström, M., Fransson, E., Hjern, A., Köhler, L., \& Wallby, T. (2014). Mental health in Swedish children living in joint physical custody and their parents' life satisfaction: A cross-sectional study. Scandinavian Journal of Psychology, 55, 433-439. https://doi.org/10.1111/sjop.12148.

Bernheimer, L. P., Weisner, T. S., \& Lowe, E. D. (2003). Impacts of children with troubles on working poor families: Mixed-method and experimental evidence. Mental Retardation, 41, 403-419. https://doi.org/10.1352/00476765(2003)41\%3c403:IOCWTO\%3e2.0.CO;2.

Boehm, J. K., \& Lyubomirsky, S. (2008). Does happiness promote career success? Journal of Career Assessment, 16, 101-116.

Bonanno, G. A. (2004). Loss, trauma, and human resilience. Have we underestimated the human capacity to thrive even after extremely aversive events? American Psychologist, 59, 20-28. https://doi.org/10.1037/0003066X.59.1.20.

Boulet, S. L., Boyle, C. A., \& Schieve, L. A. (2009). Health care use and health and functional impact of developmental disabilities among US children, 1997-2005. Archives of Pediatric \& Adolescent Medicine, 163, 1926.

Boyle, C. A., Boulet, S., Schieve, L. A., Cohen, R. A., Blumberg, S. J., Yeargin- Allsopp, M., et al. (2011). Trends in the prevalence of developmental disabilities in US children, 1997-2008. Pediatrics, 127, 
Brickman, P., \& Campbell, D. T. (1971). Hedonic relativism and planning the good society. In M. H. Appley (Ed.), Adaptation-level theory (pp. 287305). New York: Academic Press.

Burns, R. A., \& Machin, M. A. (2013). Psychological wellbeing and the diathesis-stress hypothesis model: The role of psychological functioning and quality of relations in promoting subjective well-being in a life events study. Personality and Individual Differences, 54, 321-326.

https://doi.org/10.1016/j.paid.2012.09.017.

Cacioppo, J. T., Hawkley, L. C., Kalil, A., Hughes, M. E., Waite, L., \& Thisted, R. A. (2008). Happiness and the invisible threads of social connection. The Chicago Health, Aging, and Social Relations Study. In M. Eid \& R. J. Larsen (Eds.), The science of subjective well-being (pp. 195219). New York: Guilford Press.

Campbell, R., Dworkin, E., \& Cabral, G. (2009). An ecological model of the impact of sexual assault on women's mental health. Trauma, Violence, and Abuse, 10, 225-246.

Chamberlain, K., \& Zika, S. (1990). The minor events approach to stress: Support for the use of daily hassles. British Journal of Psychology, 81, 469481.

Charles, S. T., Piazza, J. R., Mogle, J., Sliwinski, M. J., \& Almeida, D. M. (2013). The wear and tear of daily stressors on mental health. Psychological Science, 24, 733-741. https://doi.org/10.1177/0956797612462222.

Clark, A. E., Frijters, P., \& Shields, M. A. (2008). Relative income, happiness, and utility: An explanation for the Easterlin paradox and other puzzles. Journal of Economic Literature, 46, 95-144. https://doi.org/10.2307/27646948.

Coddington, R. D. (1972). The significance of life events as etiological factors in the disease of children-II. A study of a normal population. 
Journal of Psychosomatic Research, 16, 202-213.

Cohen, J. (1992). A power primer. Psychological Bulletin, 112, 155-159.

Coley, R. L., Ribar, D., \& Votruba-Drzal, E. (2011). Do children's behavior problems limit poor women's labor market success? Journal of Marriage and Family, 73, 33-45. https://doi.org/10.1111/j.1741-3737.2010.00787.x.

Coviello, L., Sohn, Y., Kramer, A. D. I., Marlow, C., Franceschetti, M., Christakis, N. A., et al. (2014). Detecting emotional contagion in massive social networks. PLoS ONE. https://doi.org/10.1371/journal.pone.0090315.

Cuijpers, P., Weitz, E., Karyotaki, E., Garber, J., \& Andersson, G. (2015). The effects of psychological treatment of maternal depression on children and parental functioning: A meta-analysis. European Child and Adolescent Psychiatry, 24, 237-245. https://doi.org/10.1007/s00787-014-0660-6.

Dearing, E. (2014). The state of research on children and families in poverty: Past, present, and future empirical avenues of promise. In K. McCartney, H. Yoshikawa, \& L. Forcier (Eds.), Improving the odds for America's children. Cambridge, MA: Harvard Education Press.

Diener, E. (1994). Subjective well-being. Psychological Bulletin, 95, 542575.

Diener, E., \& Chan, M. Y. (2011). Happy people live longer: Subjective well-being contributes to health and longevity. Applied Psychology: Health and Well-Being, 3, 1-43. https://doi.org/10.1111/j.1758-0854.2010.01045.x.

Diener, E., Diener, C., Choi, H., \& Oishi, S. (2018a). Revisiting "Most people are happy"-And discovering when they are not. Perspectives on Psychological Science, 13, 166-170. https://doi.org/10.1177/1745691618765111.

Diener, E., Emmons, R. A., Larsen, R. J., \& Griffin, S. (1985). The Satisfaction With Life Scale. Journal of Personality Assessment, 49, 71-75. 
Diener, E., \& Lucas, R. E. (1999). Personality and subjective well-being. In D. Kahneman, E. Diener, \& N. Schwarz (Eds.), Well-being: The foundations of hedonic psychology (pp. 213-229). New York: Russell-Sage.

Diener, E., Oishi, S., \& Lucas, R. E. (2003). Personality, culture, and subjective well- being: Emotional and cognitive evaluations of life. Annual Reviews of Psychology, 54, 403-425.

https://doi.org/10.1146/annurev.psych.54.101601.145056.

Diener, E., Oishi, S., \& Tay, L. (2018b). Advances in subjective well-being research. Nature Human Behavior. https://doi.org/10.1038/s41562-018-03076.

Diener, E., Pressman, S. D., Hunter, J., \& Delgadillo-Chase, D. (2017). If, why, and when subjective well-being influences health, and future needed research. Applied Psychology: Health and Well-Being, 9, 133-167. https://doi.org/10.1111/aphw.12090.

Diener, E., \& Seligman, M. E. P. (2002). Very happy people. Psychological Science, 13, 81-84.

Diener, E., \& Tay, L. (2015). Subjective well-being and human welfare around the world as reflected in the Gallup World Poll. International Journal of Psychology, 50, 135-149. https://doi.org/10.1002/ijop.12136.

Dunn, E., \& Norton, M. (2013). Happy money: The science of happier spending. London: Simon and Schuster.

Dyrdal, G. M., Røysamb, E., Nes, R. B., \& Vittersø, J. (2011). Can a happy relationship predict a happy life? A population-based study of maternal wellbeing during the life transition of pregnancy, infancy, and toddlerhood. Journal of Happiness Studies, 12, 947-962. https://doi.org/10.1007/s10902010-9238-2.

Easterlin, R. A. (1974). Does economic growth improve the human lot? In P. A. David \& M. W. Reder (Eds.), Nations and households in economic growth (pp. 89-125). New York: Academic Press. 
Easterlin, R. A. (2013). Happiness and economic growth: The evidence. Discussion Paper Series, Forschungsinstitut zur Zukunft der Arbeit, No. 7187. http://hdl.handle.net/10419/69363.

Easterlin, R. A., McVey, L. A., Switek, M., Sawangfa, O., \& Zweig, J. S. (2010). The happiness-Income paradox revisited. PNAS, 107, 2246322468. https://doi.org/10.1073/pnas.1015962107.

Fisak, B., Holderfield, K. G., Douglas-Osborn, E., \& Cartwright-Hatton, S. (2012). What do parents worry about? Examination of the construct of parent worry and the relation to parent and child anxiety. Behavioural and Cognitive Psychotherapy, 40, 542-557. https://doi.org/10.1017/S1352465812000410.

Fredrickson, B. L. (2013). Positive emotions broaden and build. In D. Patricia \& P. Ashby (Eds.), Advances in experimental social psychology (Vol. 47, pp. 1-53). Cambridge: Academic Press.

Glover, V. (2011). Annual research review: Prenatal stress and the origins of psychopathology: an evolutionary perspective. The Journal of Child Psychology and Psychiatry, 52, 356-367. https://doi.org/10.1111/j.14697610.2011.02371.x.

Glover, V. (2014). Maternal depression, anxiety and stress during pregnancy and child outcome; what needs to be done. Best Practice \& Research Clinical Obstetrics and Gynaecology, 28, 25-35. https://doi.org/10.1016/j.bpobgyn.2013.08.017.

Goldman-Mellor, S. J., Saxton, K. B., \& Catalano, R. C. (2010). Economic contraction and mental health. A review of the evidence, 1990-2009. International Journal of Mental Health, 39, 6-31. https://doi.org/10.2753/IMH0020-7411390201.

Goldsmith, A. H., Veum, J. R., \& Darity, W. (1996). The impact of labor force history on self-esteem and its component parts, anxiety, alienation and depression. Journal of Economic Psychology, 17, 183-220. 
Goodman, S. H. (2007). Depression in mothers. The Annual Review of Clinical Psychology, 3, 107-135.

https://doi.org/10.1146/annurev.clinpsy.3.022806.091401.

Graham, C., Chattopadhyay, S., \& Picon, M. (2010). The Easterlin paradox and other paradoxes: Why both sides of the debate may be correct. In E. Diener, J. F. Helliwell, \& D. Kahneman (Eds.), International differences in well-being (pp. 247-288). Oxford: Oxford University Press.

Green, F. (2011). Unpacking the misery multiplier: How employability, modifies the impacts of unemployment and job insecurity on life satisfaction and mental health. Journal of Health Economics, 30, 265-276. https://doi.org/10.1016/j.jhealeco.2010.12.005.

Halfon, N., Houtrow, A., Larson, K., \& Newacheck, P. W. (2012). The changing landscape of disability in childhood. The Future of Children, 22, $13-42$.

Hauge, L. J., Nes, R. B., Kornstad, T., Kristensen, P., Irgens, L. M., Landolt, M. A., et al. (2015). Maternal sick leave due to psychiatric disorders following the birth of a child with special health care needs. Journal of Pediatric Psychology, 40(8), 804-813.

Headey, B. (2013). Set-point theory may now need replacing: Death of a paradigm? In S. David, I. Boniwell, \& A. C. Ayers (Eds.), The Oxford handbook of happiness (pp. 887-900). Oxford: Oxford University Press.

Headey, B., \& Wearing, A. (1989). Personality, life events, and subjective well-being: Toward a dynamic equilibrium model. Journal of Personality and Social Psychology, 57, 731-739.

Hobbs, D. F. (1968). Transition to parenthood: A replication and an extension. Journal of Marriage and Family, 30, 413-417.

Holmes, E. K., Erickson, J. J., \& Hill, E. J. (2012). Doing what she thinks is best: Maternal psychological wellbeing and attaining desired work situations. Human Relations, 65, 501-522. 
https://doi.org/10.1177/0018726711431351.

Holt-Lunstad, J., Smith, T. B., \& Layton, J. B. (2010). Social relationships and mortality risk: A meta-analytic review. PLoS Medicine, 7(2-20), e1000316. https://doi.org/10.1371/journal.pmed.1000316.

Howell, R. T., Kern, M. L., \& Lyubomirksy, S. (2007). Health benefits: Meta- analytically determining the impact of well-being on objective health outcomes. Health Psychology Review, 1, 83-136.

Infurna, F. J., Gerstorf, D., Ram, N., \& Heckhausen, J. (2016). Analytic strategies for the study of adaptation to major life events: making the most of large-scale longitudinal surveys. In Methodological issues of longitudinal surveys (pp. 19-35). Wiesbaden: Springer Fachmedien Wiesbaden.

Infurna, F. J., Wiest, M., Gerstorf, D., Ram, N., Schupp, J., Wagner, G. G., et al. (2016b). Changes in life satisfaction when losing one's spouse: individual differences in anticipation, reaction, adaptation and longevity in the German Socio-economic Panel Study (SOEP). Ageing \& Society. https://doi.org/10.1017/S0144686x15001543.

Institute for Health Metrics and Evaluation (IHME). (2016). Norway: State of the Nation's Health: Findings from the global burden of disease. Seattle, WA: IHME.

Kahneman, D., \& Deaton, A. (2010). High income improves evaluation of life but not emotional well-being. Proceedings of the National Academy of Sciences in the United States of America (PNAS), 107, 16489-16493. https://doi.org/10.1073/pnas.1011492107.

Kanner, A. D., Coyne, J. C., Schaefer, C., \& Lazarus, R. S. (1981). Comparison of two modes of stress measurement: Daily hassles and uplifts versus major life events. Journal of Behavioral Medicine, 4, 1-39. https://doi.org/10.1007/BF00844845.

Kapteyn, A., Lee, J., Tassot, C., Vonkova, H., \& Zamarro, G. (2015). Dimensions of subjective well-being. Social Indicators Research, 123, 625- 
660.

Lazarus, R. S. (1986). Puzzles in the study of daily hassles. In R. K. Silbereisen, K. Eyferth, \& G. Rudinger (Eds.), Development as action in context. Berlin, Heidelberg: Springer. https://doi.org/10.1007/978-3-66202475-13.

Lucas, R. E. (2005). Time does not heal all wounds: A longitudinal study of reaction and adaptation to divorce. Psychological Science, 16, 945-950.

Lucas, R. E. (2007). Long-term disability is associated with lasting changes in subjective well-being: Evidence from two nationally representative longitudinal studies. Journal of Personality and Social Psychology, 92, 717730 .

Lucas, R. E. (2018). Reevaluating the strengths and weaknesses of selfreport measures of subjective well-being. In E. Diener, S. Oishi, \& L. Tay (Eds.), Handbook of subjective well-being. Noba Scholar Handbook series: Subjective well-being. Salt Lake City, UT: DEF Publishers.

Lucas, R. E., \& Clark, A. E. (2006). Do people really adapt to marriage?. Journal of Happiness Studies, 7, 405-426.

Lucas, R. E., Clark, A. E., Georgellis, Y., \& Diener, E. (2004). Unemployment alters the set point for life satisfaction. Psychological Science, 15, 8-13.

Lucas, R. E., \& Schimmack, U. (2009). Income and well-being: How big is the gap between the rich and the poor? Journal of Research in Personality, $43,75-78$.

Luhmann, M., Hofmann, W., Eid, M., \& Lucas, R. E. (2011). Subjective well-being and adaptation to life events: A meta-analysis. Journal of Personality and Social Psychology. https://doi.org/10.1037/a0025948.

Magnus, P., Birke, C., Vejrup, K., Haugan, A., Alsaker, E., Daltveit, A. K., et al. (2016). Cohort profile update: The Norwegian Mother and Child Cohort 
Study (MoBa). International Journal of Epidemiology, 45, 382-388.

Maroto, M. L. (2015). Pathways into bankruptcy: Accumulating disadvantage and the consequences of adverse life events. Sociological Inquiry, 85, 183-216. https://doi.org/10.1111/soin.12073.

Marum, G., Clench-Aas, J., Nes, R. B., \& Raanaas, R. K. (2013). The relationship between negative life events, psychological distress, and life satisfaction: A population-based study. Quality of Life Research, 23, 1-11.

McMunn, A., Kelly, Y., Cable, N., \& Bartley, M. (2010). Maternal employment and child socio-emotional behaviour: Longitudinal evidence from the millennium cohort study. Journal of Epidemiology and Community Health, 64, A32-A33. https://doi.org/10.1136/jech.2010.109553.

Messing, J. T., Thaller, J., \& Bagwell, M. (2014). Factors related to sexual abuse and forced sex in a sample of women experiencing police-involved intimate partner violence. Health and Social Work, 39, 181-191. https://doi.org/10.1093/hsw/hlu026.

Miller, B. C., \& Sollie, D. L. (1980). Family stress, copying and adaptation. Family Relations, 29, 459-465.

Murray, C. J. L., \& Lopez, A. D. (1997). Alternative projections of mortality and disease by cause 1990-2020: Global burden of disease study. The Lancet, 349, 1498-1504. https://doi.org/10.1016/S0140-6736(96)07492-2.

Næss, S., Blekesaune, M., \& Jakobsson, N. (2015). Marital transitions and life satisfaction. Evidence from longitudinal data from Norway. Acta Sociologica, 58, 63-78. https://doi.org/10.1177/0001699314563841.

Nes, R. B., Hauge, L. J., Kornstad, T., Kristensen, P., Landolt, M. A., Eskedal, L. T., et al. (2014a). The impact of child behaviour problems on maternal employment: A longitudinal cohort study. Journal of Family and Economic Issues, 35, 351-361.

Nes, R. B., Røysamb, E., Hauge, L. J., Kornstad, T., Landolt, M., Irgens, L., 
et al. (2014b). Maternal well-being and psychological distress: A prospective longitudinal study of mothers giving birth to children with congenital anomalies. Developmental Psychology, 50, 1827-1839.

Nes, R. B., Røysamb, E., Tambs, K., Harris, J. R., \& Reichborn-Kjennerud, T. (2006). Subjective well-being: Genetic and environmental contributions to stability and change. Psychological Medicine, 36, 1033-1042.

Nielsen, M. B., Magerøy, N., Gjerstad, J., \& Einarsen, S. (2014). Workplace bullying and subsequent health problems. Tidsskrift for Den Norske Laegeforening: Tidsskrift for Praktisk Medisin, 134, 1233-1238. https://doi.org/10.4045/tidsskr.13.0880.

North, R. J., Holahan, C. J., Moos, R. H., \& Cronkite, R. C. (2008). Family support, family income, and happiness: A 10-year perspective. Journal of Family Psychology, 22, 475-483.

Norwegian Mother and Child Cohort Study, Revised Protocol (2010). Norwegian Institute of Public Health.

O'Hara, M. W., \& Wisner, K. L. (2014). Perinatal mental illness: Definition, description, and aetiology. Best Practice \& Research Clinical Obstetrics \& Gynaecology, 28, 3-12. https://doi.org/10.1016/j.bpobgyn.2013.09.002.

Oishi, S. (2012). Relational wealth. In The psychological wealth of nations: Do happy people make a happy society? (pp 57-72). West Sussex: Wiley.

Pavot, W., \& Diener, E. (2008). The Satisfaction With Life Scale and the emerging construct of life satisfaction. The Journal of Positive Psychology, $3,137-152$.

Pearlin, L. I., Menaghan, E. G., Lieberman, M. A., \& Mullan, J. T. (1981). The stress process. Journal of Health and Social Behavior, 22, 337-356.

Powdthavee, N. (2008). Putting a price tag on friends, relatives, and neighbours: Using surveys of life satisfaction to value social relationships. The Journal of Socio- Economics, 37, 1459-1480. 
Reneflot, A., \& Evensen, M. (2014). Unemployment and psychological distress among young adults in the Nordic countries: A review of the literature. International Journal of Social Welfare, 23, 3-15. https://doi.org/10.1111/ijsw.12000.

Rhoades, G. K., Dush, C. M. K., Atkins, D. C., Stanley, S. M., \& Markman, H. J. (2011). Breaking up is hard to do: The impact of unmarried relationship dissolution on mental health and life satisfaction. Journal of Family Psychology, 25, 366-374.

Robinson, K., Kennedy, N., \& Harmon, D. (2012). Happiness: A review of evidence relevant to occupational science. Journal of Occupational Science, $19,150-164$.

Røsand, G. M. B., Slinning, K., Eberhart-Gran, M., Røysamb, E., \& Tambs, K. (2012). The buffering effect of relationship satisfaction on emotional distress in couples. BioMed Central Public Health, 12, 66-79.

Rosenzweig, J. M., Brennan, E. M., Huffstutter, K., \& Bradley, J. R. (2008). Child care and employed parents of children with emotional or behavioral disorders. Journal of Emotional and Behavioral Disorders, 16(2), 78-89. https://doi.org/10.1177/1063426607312538.

Roy, R. N., Schumm, W. R., \& Britt, S. L. (2014). Transition to Parenthood. New York, NY: Springer. https://doi.org/10.1007/978-1-4614-7768-6.

Seery, M. D., Holman, E. A., \& Silver, R. C. (2010). Whatever does not kill us: Cumulative lifetime adversity, vulnerability, and resilience. Journal of Personality and Social Psychology, 99, 1025-1041.

Sitnik, S. L., Masyn, K., Ontai, L. L., \& Conger, K. J. (2016). Mothers'physical illness in one- and two-parent families. Journal of Family Issues, 37, 902-920. https://doi.org/10.1177/0192513X14536563.

Sonnentag, S., Unger, D., \& Nägel, I. J. (2013). Workplace conflict and employee well-being: The moderating role of detachment from work during off-job time. International Journal of Conflict Management, 24, 166-183. 
https://doi.org/10.1108/10444061311316780.

Steptoe, A., Deaton, A., \& Stone, A. A. (2015). Subjective wellbeing, health, and ageing. The Lancet, 385, 640-648. https://doi.org/10.1016/S01406736(13)61489-0.

Stickler, G. B., Salter, M., Broughton, D. D., \& Alario, A. (1991). Parents 'worries about children compared to actual risks. Clinical Pediatrics, 30, $522-528$.

Suarez, E. B., Lafrenière, G., \& Harrison, J. (2016). Scoping review of interventions supporting mothers with mental illness: Key outcomes and challenges. Community Mental Health Journal, 52, 927-936. https://doi.org/10.1007/s10597-016-0037-z.

Taylor, S. (2012). Transformation through suffering: A study of individuals who have experienced positive psychological transformation following periods of intense turmoil. Journal of Humanistic Psychology, 52, 30-52.

Teigen, K. H., \& Glad, K. A. (2011). "It Could have been Much Worse": From travelers' accounts of two natural disasters. Scandinavian Journal of Hospitality and Tourism, 11, 237-249. https://doi.org/10.1080/15022250.2011.606610.

Thoits, P. A. (1983). Multiple identities and psychological well-being: A reformulation and test of the social isolation hypothesis. American Sociological Review, 48, 174-187.

Umberson, D., \& Montez, J. K. (2010). Social relationships and health: A flashpoint for health policy. Journal of Health and Social Behavior, 51, 5466. https://doi.org/10.1177/0022146510383501.

Usdansky, M., Gordon, R., Wang, X., \& Gluzman, A. (2012). Depression risk among mothers of young children: The role of employment preferences, labor force status and job quality. Journal of Family and Economic Issues, 33(1), 83-94. https://doi.org/10.1007/s10834-011-9260-5. 
Walzer, S. (1996). Thinking about the baby: Gender and division of infant care. Social Problems, 43, 219-234. https://doi.org/10.2307/3096999.

Zachrisson, H. D., \& Dearing, E. (2015). Family income dynamics, early childhood education and care, and early child behavior problems in Norway. Child Development, 86, 425-440.

1 The reported sample sizes refer to $\mathrm{N}$ of pregnancies. However, some women were recruited more than once (i.e., when expecting a new baby subsequent to the initial pregnancy), thereby allowing for inclusion of siblings among the children. Thus, the number of women included was lower than the number of pregnancies. We chose to include the multiple entries of women, as this strategy would retain all available information. Although some women were included twice, each data entry refers to different time periods. We also reran the analyses with only single entries for each woman; the findings and conclusions remained the same as those reported here. 